\title{
Simplified SEE Sensitivity Screening for COTS Components in Space
}

\author{
Rubén García Alía, Markus Brugger, Eamonn Daly, Salvatore Danzeca, Véronique Ferlet-Cavrois, Rémi Gaillard, \\ Julien Mekki, Christian Poivey, Ali Zadeh
}

\begin{abstract}
We introduce an approach aimed at pre-screening COTS components according to their SEE sensitivity for space missions in which a complete characterization of their individual response to protons and heavy ions is not feasible due to cost and time constraints. The method is applied to a set of SRAM memories for SEU and SEL and the resulting expected SEE rates are compared with traditional approaches and in-flight data for a LEO polar and geostationary orbit. Despite the limitations related to components with high-LET threshold and thick sensitive volumes, we conclude that the proposed method can be an efficient means of rejecting highly sensitive components or lots and performing the complete characterization only on passing devices.
\end{abstract}

Index Terms-Radiation hardness assurance methodology, single-event effect (SEE), single-event latchup (SEL), CHARM radiation facility, FLUKA, Monte Carlo methods

\section{INTRODUCTION}

Despite the high risk related to heavy-ion (HI) induced Single Event Effects (SEEs) in the space radiation environment, thoroughly testing all mission components through HI ground experiments is often too costly and impractical, especially for low-budget cubesat missions. Therefore, different efforts have been carried out in order to use more accessible proton data as a means of bounding the HI sensitivity ([1] and references therein). Such approaches are based on the fact that, in their nuclear interactions with $\mathrm{Si}$ atoms in and near the device's Sensitive Volumes (SVs), protons (or more generally hadrons) will generate fragments and recoils with LET values up to $15 \mathrm{MeVcm}^{2} / \mathrm{mg}$, therefore covering a large fraction of the Galactic Cosmic Ray (GCR) LET spectrum. However, establishing the link between the experimental context and the environmental conditions is challenging due to the complex charge, energy and angular distributions of the nuclear fragments. Therefore such approaches strongly rely on the Monte Carlo simulation of the fragment production, which can be represented and correlated with the application case in different phase spaces.

Similarly to what was initially proposed in [2] and recently developed in [1], [3] we will represent and compare the various conditions in the deposited energy phase space through the definition of the volume-equivalent LET as the deposited

R. García Alía (ruben.garcia.alia@cern.ch), M. Brugger, and S. Danzeca are with CERN, CH-1211, Genève, Switzerland.

E. Day, V. Ferlet-Cavrois, C. Poivey and A. Zadeh are with the European Space Agency, ESTEC, 2200 AG Noordwijk, The Netherlands.

J. Mekki is with CNES, 18 av Edouard Belin, 31055, Toulouse, France.

R. Gaillard is a consultant in 78730 Saint-Arnoult, France. energy divided by the SV thickness. In this way the limitations associated to the LET metric for nuclear fragments are overcome, the main one being its strong variability throughout the path of the fragment in the SV (who's dimensions however need to be defined). As we will later show, in the case of large SVs and a high LET threshold (i.e. being sensitive mainly to heavy fragments with short ranges) describing the experimental hadronic environment through the LET of the produced fragments can lead to a significant overestimation of the robustness of the tested component. This is due to the fact that the initial fragment LET times the SV thickness can be notably larger than the energy actually deposited.

The most recent effort of extracting the HI cross section from proton test data [1] relies on Monte Carlo simulations of the proton deposited energy distribution for the selected SV dimensions and determination of the optimal heavy ion Weibull fit parameters through a maximum likelihood analysis. The approach we present here follows a similar energy deposition metric for the hadronic experimental case, but instead of using the calculated distribution(s) to determine the best fit to a Weibull HI response, establishes the link with the space environment by directly comparing the respective energy deposition distributions. The conclusion is that, for an SV thickness up to $2 \mu \mathrm{m}$ and a relatively large volume-equivalent LET interval (roughly $1-10 \mathrm{MeVcm}^{2} / \mathrm{mg}$ ) the ratio between the experimental and application environments remains within a factor of 3 , and therefore a constant proportionality factor can be established and exploited to estimate the HI space environment SEE rate based on single hadron measurements. The approach is introduced and applied to a set of five different components on-board two ESA SEE experiments, and the estimated results are compared with the in-flight data retrieving an agreement level similar to what is obtained through traditional SEE rate prediction approaches, involving a much more intensive experimental effort.

In addition, we analyze the dependency of the obtained proportionality factors linking experimental and application conditions with the hadron field and the SV dimensions and surrounding materials, underlining the implications of the results on the limitation of the approach. Related to this, ground tests are presented not only for standard monoenergetic proton facilities but also for the CHARM mixed-field facility at CERN [4]-[6], which in terms of energy deposition distributions has the advantage of enabling the possibility of testing in hadron environments that are more energetic than standard proton facilities. As will be detailed later on, another practical advantage of the CHARM facility when compared 
to monoenergetic proton facilities is that of enabling the possibility of testing a large number of components in parallel or even performing full system tests.

The paper is structured as follows: the CHARM facility and the parametrization of its SEE-relevant environment is briefly described in Section II. The proposed approach is detailed in Section III and applied to a set of test and space environments for a specific SEL-like SV geometry. In Section IV the approach is applied to a set of commercial SRAM components tested in both monoenergetic facilities and at CHARM and compared to in-flight data from two ESA experiments as well as with results extracted through the standard qualification approach. The dependency of the applied proportionality factors with the model inputs is discussed along with the associated hardness overestimation risk. The associated Radiation Hardness Assurance (RHA) implications are covered in Section $\mathrm{V}$ with the conclusions of the proposed approach and its application described in Section VI.

\section{HEH FORMALISM AT CHARM}

As described in more detail in [4]-[6] CHARM is a mixedfield facility reproducing the radiation environment in a highenergy accelerator through the interaction of a $24 \mathrm{GeV}$ proton beam with a $50 \mathrm{~cm}$ copper target. A broad range of test locations and shielding configurations are available yielding a set of particle energy spectra that mimic not only various accelerator cases (e.g. LHC tunnel, shielded alcoves [7]) but also other application contexts of interest such as the atmospheric neutron or trapped proton space environments. A horizontal cut of the CHARM irradiation area model can be seen in Fig. 1 in which the movable shielding is placed inside. The positions with the red labels from 1 to 13 represent the different available test locations.

For SEE purposes, the complex mixed radiation field at CHARM is described through the High Energy Hadron (HEH) fluence, defined as the fluence of hadrons with an energy above $20 \mathrm{MeV}$ [8] and extracted through benchmarked Monte Carlo calculations using FLUKA [9]-[11]. The definition is based on the assumption that the different hadrons present in the CHARM field (mainly neutrons, protons and pions and in proportions that depend on the specific test location and shielding configuration) are equally efficient in inducing SEEs above $20 \mathrm{MeV}$, whereas below this energy they are irrelevant in terms of SEE induction. Therefore, the CHARM SEE cross sections are referred to as $\mathrm{HEH}$ cross sections $\left(\sigma_{\mathrm{HEH}}\right)$ and defined as the number of SEEs divided by the HEH fluence.

CHARM results presented in this paper were collected in position 4, both without and with shielding. The associated test conditions are labeled as $\operatorname{CHARM}(4)$ and $\operatorname{CHARM}\left(4^{*}\right)$ respectively.

When considering SEE tests for space applications, the trapped proton belt environment [12] characteristic of LEO orbits can be accurately reproduced at CHARM in terms of the hadron energy spectrum as can be seen in Fig. 2 where the normalized differential spectra are shown for the ProbaII orbit and several test locations at CHARM. The associated acceleration factor for the ground testing is roughly $5 \times 10^{2}$.

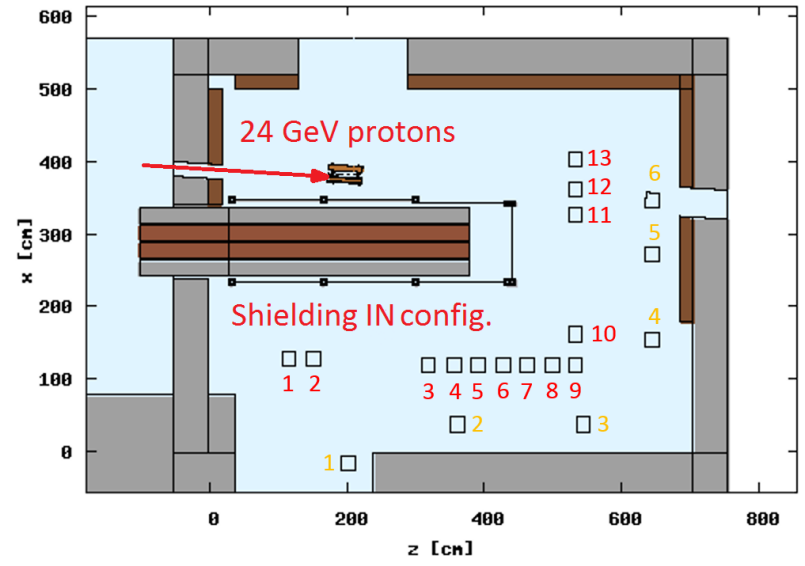

Fig. 1: Horizontal cut of the FLUKA geometry of the CHARM facility.

Therefore, in general the associated trapped proton SEE rate can be derived as the product of the HEH cross section at CHARM and the HEH flux in orbit (similarly to how the neutron flux above $10 \mathrm{MeV}$ is used to correlate ground level SEE rates with spallation neutron facility test results).

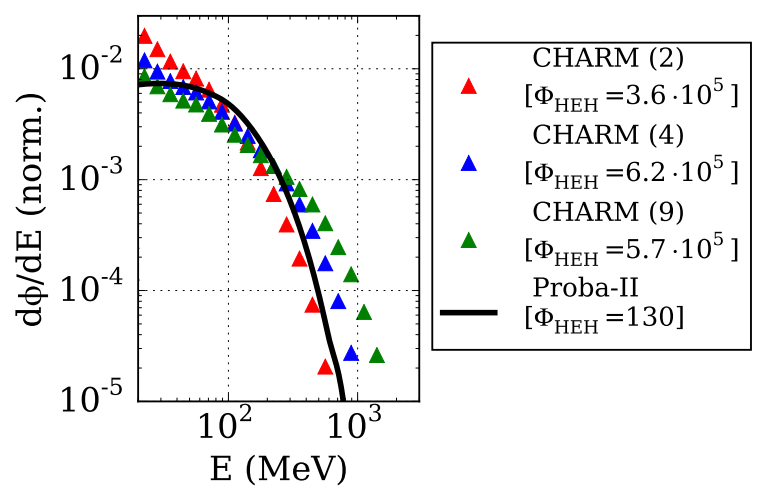

Fig. 2: Differential HEH spectrum for the Proba-II orbit (100 mils aluminum shielding) as extracted using CREME96 [13] and different test locations at CHARM as obtained using FLUKA. Results are normalized to the HEH flux $\Phi_{\mathrm{HEH}}$ which is shown in the legend in units of $\mathrm{cm}^{-2} \mathrm{~s}^{-1}$.

It is to be noted however that in addition to the spectral hardness, other considerations need to be taken into account when comparing the CHARM and trapped proton belt environments, such as:

- the composition of the HEH flux, which in the LEO case is purely protons and for CHARM will consist of protons, pions and neutrons in a proportion that depends on the test configuration (e.g. 70\% neutrons, $14 \%$ protons and $16 \%$ pions for test location 4 , no shielding);

- contributions not taken into account in the HEH formalism, such as neutrons below $20 \mathrm{MeV}$ (including thermals) [8] or direct ionization from singly charged particles in the $\mathrm{MeV}$ range [14], and which can have a different relative impact at CHARM and in a LEO orbit;

- the possible contribution of Total Ionizing Dose (TID) 
to the degradation of the component or the SEE cross section being measured.

As to what concerns this final point, the dose-to-HEH fluence can be compared for both environments. Using the OMERE tool [15], an annual TID value of $3.16 \mathrm{krad}(\mathrm{Si})$ was obtained for the Proba-II orbit and 100 mils of aluminum shielding. When divided by the annual HEH fluence for the same orbit $\left(4.1 \times 10^{9} \mathrm{~cm}^{-2} \mathrm{y}^{-1}\right)$ this yields a dose-to-fluence ratio of $770 \mathrm{rad}(\mathrm{Si})$ per $10^{9} \mathrm{HEH} / \mathrm{cm}^{2}$. At CHARM(4), the calculated dose-to-fluence value is $62 \mathrm{rad}(\mathrm{Si})$ per $10^{9} \mathrm{HEH} / \mathrm{cm}^{2}$, therefore roughly a factor of 10 smaller and similar to the value for $200 \mathrm{MeV}$ protons. Consequently, similarly to the case of standard proton testing, if TID effects are of interest, the difference in the dose-to-fluence ratio needs to be taken into account.

\section{VOLUME-EQUIVALENT LET APPROACH - VELA}

The volume-equivalent LET approach (VELA) we propose here is based on the representation of a radiation environment through its energy deposition distribution in a certain SV, divided by the SV thickness. Therefore, the metric has units of LET, however it is to be born in mind that it is no longer an intrinsic property of the particles (such as e.g. their production LET) but rather a value related to the energy deposition in a certain volume, thus also depending on the SV dimensions and surrounding materials.

In the energy deposition phase space, the SEE rate can be defined as the convolution of the environmental differential flux for the considered application $\phi_{\mathrm{app}}$ and cross section $\sigma$, both as a function of volume-equivalent LET $\left(\operatorname{LET}_{\mathrm{V}}\right)$. Moreover, if we assume that the cross section follows a step function at a certain $\operatorname{LET}_{\mathrm{V}}=\mathrm{LET}_{\mathrm{V}}^{*}$, the expression can be simplified as shown in Eq. 1 where $\Phi_{\text {app }}\left(>L T_{v}^{*}\right)$ is the integral flux of particles depositing an equivalent LET value larger than $\mathrm{LET}_{\mathrm{v}}^{*}$ in the application environment.

$$
\mathrm{R}=\int \phi_{\mathrm{app}}\left(\mathrm{LET}_{\mathrm{V}}\right) \sigma\left(\mathrm{LET}_{\mathrm{V}}\right) \mathrm{dLET}_{\mathrm{V}}=\Phi_{\mathrm{app}}\left(>\mathrm{LET}_{\mathrm{V}}^{*}\right) \sigma\left(\mathrm{LET}_{\mathrm{V}}^{*}\right)
$$

The cross section in Eq. 1 is experimentally determined by dividing the number of counts $\mathrm{N}$ by the associated experimental fluence above the considered volume-equivalent LET value, $\Phi_{\exp }\left(>\mathrm{LET}_{\mathrm{v}}^{*}\right)$. However, when considering a hadron environment, one can multiply and divide the expression by the associated $\mathrm{HEH}$ fluence value $\Phi_{\mathrm{HEH}}$ as shown in Eq. 2, which when combined with Eq. 1 yields the expression shown in Eq. 3 relating the application SEE rate $\mathrm{R}$ with the experimental $\mathrm{HEH}$ cross section through a proportionally factor $\alpha$ having flux units. The $\alpha$-factor corresponds to the event rate per unit surface with a volume-equivalent LET value above threshold in the application environment times the ratio between the experimental HEH fluence and fluence above the same volume-equivalent LET threshold.

$$
\sigma\left(\mathrm{LET}_{\mathrm{v}}^{*}\right)=\frac{\mathrm{N}}{\Phi_{\exp }\left(>\mathrm{LET}_{\mathrm{v}}^{*}\right)}=\frac{\Phi_{\mathrm{HEH}}}{\Phi_{\exp }\left(>\mathrm{LET}_{\mathrm{v}}^{*}\right)} \cdot \sigma_{\mathrm{HEH}}
$$

$$
\mathrm{R}=\Phi_{\mathrm{app}}\left(>\mathrm{LET}_{\mathrm{v}}^{*}\right) \cdot \frac{\Phi_{\mathrm{HEH}}}{\Phi_{\exp }\left(>\mathrm{LET}_{\mathrm{v}}^{*}\right)} \cdot \sigma_{\mathrm{HEH}}=\alpha\left(\mathrm{LET}_{\mathrm{v}}^{*}\right) \cdot \sigma_{\mathrm{HEH}}
$$

The strongest assumption in the formalism introduced above is that of assigning a step function to $\sigma\left(\mathrm{LET}_{\mathrm{v}}^{*}\right)$ whereas in general a Weibull-like dependency exists according to which the cross section progressively increases with LET until reaching a saturation value. As explained in [1], in order to extract the parameters of such a function from a set of hadron experimental data, a maximum likelihood analysis needs to be performed with 4 degrees of freedom, which implies that different sets of values might result is similarly satisfactory fits potentially yielding very different SEE rates when convoluted with the environmental (e.g. space) LET spectrum.

The step response function assumption is justified and discussed in the application of the approach, which is carried out as follows: a generic SV and surrounding geometry is selected for the SEE of interest. Provided that typically a limited amount of information is available on the specific component layout, we consider a pure silicon geometry with SV dimensions based on available information for the concerned SEE and technology.

Once we have defined the simple geometry model of the SV and its relevant surroundings, we perform Monte Carlo simulations of the energy deposition distribution in the application and experimental contexts. In order to illustrate the method, we consider two space environments: a $800 \mathrm{~km} 98^{\circ}$ Low-Earth Orbit (LEO) and a near-Earth interplanetary space environment (e.g. geostationary orbit, GEO). For SEL the SV dimensions are considered as $20 \times 4 \times 2 \mu \mathrm{m}^{3}$ according to laser studies of similar technologies [16]. Indeed, $2 \mu \mathrm{m}$ is the standard SV thickness used in recent in-flight SEL rate studies [17], [18] however as will be later discussed should not be applied as a general reference value. For SEU, we assume an $\mathrm{SV}$ volume of $3 \times 3 \times 0.5 \mu \mathrm{m}^{3}$ according to the cell size of the considered SRAM and the associated estimated charge collection depth. In both cases, a Back-End-Of-Line (BEOL) of $5 \mu \mathrm{m}$ of silicon is considered. An analysis of the impact of the SV depth and surrounding materials on the VELA approach proportionality factor $\alpha$ will be shown in Section IV-D with a focus on the risk of overestimating the radiation hardness of components with larger SVs.

We use CREME96 to generate the environments using the CREME96 GCR version [13], a Solar-quiet mode (1977), ions between $Z=1$ and $Z=92,100$ mils of aluminum shielding and both an $800 \mathrm{~km}, 98^{\circ}$ orbit and a Geosynchronous/near-Earth interplanetary environment; and CRÈME MC [19] to transport the environments through the defined geometry, retrieving as an output the corresponding SEE rate as a function of the threshold volume-equivalent LET.

Concerning the experimental case, we consider both a 230 $\mathrm{MeV}$ proton beam and the CHARM HEH environment (i.e. only hadrons above $20 \mathrm{MeV}$ are simulated) for position 4 with and without shielding. The relevant FLUKA-generated $\mathrm{HEH}$ spectra (protons, neutrons and pions above $20 \mathrm{MeV}$ ) are transported through the model geometry again using FLUKA and the corresponding energy deposition distributions are 
extracted, integrated and normalized in order to yield the corresponding cross section as a function of volume-equivalent LET. The resulting application SEE rate and experimental cross sections are shown in Fig. 3 normalized to the respective SV surface and compared to a trapped proton LEO environment (i.e. that of Proba-II). The curves are represented in two different y-axes: SEE rate for the in-flight case, and HEH cross section for the ground-level test scenarios. As will be detailed later, the proportionality factor amongst the axes (defined as $\alpha$ in Eq. 3) is selected in such a way that the experimental cross section and in-flight SEE rate coincide for a given volumeequivalent LET value.

As can be seen, all three environments considered (two experimental hadronic cases and the LEO trapped-proton belt) yield a very similar behavior as a function of the volumeequivalent LET in the range above $\sim 1 \mathrm{MeVcm}^{2} / \mathrm{mg}$. This is due to the fact that despite the differences in the considered environments (monoenergetic protons, a proton spectrum for the LEO orbit and a set of hadron spectra for the CHARM environment) the likelihood and fragment properties of the associated nuclear events are fairly independent of the hadron species and energy. The respective distributions can then be used to extract the $\alpha$-factors as defined in Eq. 3 and therefore relating the application SEE rate with the experimental $\mathrm{HEH}$ cross section. This has to be done for a specific volumeequivalent LET value, however as is observed $\alpha$ hardly depends on $\mathrm{LET}_{\mathrm{V}}$ and therefore can be applied to cases with a very broad range of LET threshold values. This is also the reason why the step function approximation in Eq. 1 generally holds: despite the fact the response function actually has a dependency with LET, the ratio between the application and experimental distributions is constant over the relevant LET interval, therefore the proportionality factor will only weakly depend on the actual LET response.

In order to define $\alpha$ for a given pair of application and experimental cases, we arbitrarily select the value relating both quantities for a volume-equivalent LET threshold value of $3 \mathrm{MeVcm}^{2} / \mathrm{mg}$.

In addition, it is to be noted that the fact that the ProbaII curve in Fig. 3 below $\sim 1 \mathrm{MeVcm}^{2} / \mathrm{mg}$ is significantly larger than the CHARM one is due to the impact of direct ionization from protons, which is suppressed in the mixedfield case due to the fact that only hadrons above $20 \mathrm{MeV}$ are considered. An analysis about the expected direct ionization contribution in the high-energy accelerator mixed-field and CHARM experimental facility can be found in [20].

In a similar way we apply the approach to the same experimental cases and a near-Earth (GEO) interplanetary GCR application environment. Both outputs (SEE rate for the space case and cross section for experimental conditions) are again plotted in two separate $y$-axes both as a function of $\mathrm{LET}_{\mathrm{v}}$ as shown in Fig. 4. As can be seen, the experimental and application SEL cross section/rate follow a comparable $\mathrm{LET}_{\mathrm{V}}$ trend in the $\sim 1-10 \mathrm{MeVcm}^{2} / \mathrm{mg}$ range. Despite the genuinely diverse radiation fields (GCR ions versus highenergy hadrons), when represented in the volume-equivalent LET phase space, the environments are comparable in a relatively wide LET range. This again justifies the use of a

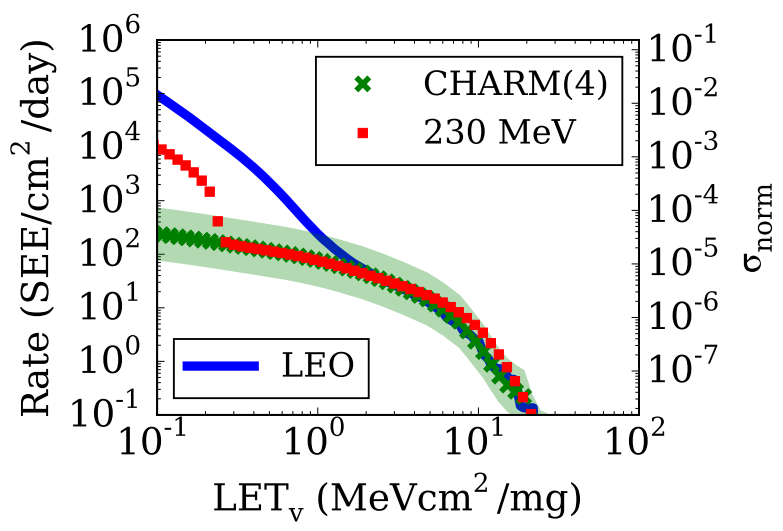

Fig. 3: Simulated SEE rate and experimental cross section as a function of the volume-equivalent LET for the considered SEL SV. The shaded region corresponds to a factor of 3 margin with respect to the CHARM curve.

single $\alpha$ value to relate both cases, however in this occasion there is a certain dependency of $\alpha$ with $\mathrm{LET}_{\mathrm{v}}$, especially for values above $\sim 10 \mathrm{MeVcm}^{2} / \mathrm{mg}$ for which applying an $\alpha$ factor extracted from the $1-10 \mathrm{MeVcm}^{2} / \mathrm{mg}$ interval can lead to a significant underestimation of the environmental SEE rate. The implications of such possibility on the Radiation Hardness Assurance (RHA) of the VELA method will be discussed in Section V.

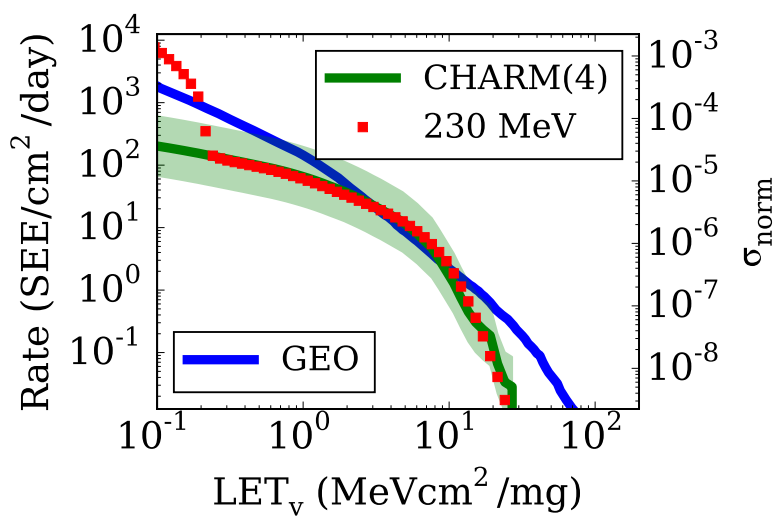

Fig. 4: Simulated SEE rate and experimental cross section as a function of the volume-equivalent LET for the considered SEL SV. The shaded region corresponds to a factor of 3 margin with respect to the CHARM curve.

The application SEE rate and experimental cross sections in Figs. 3 and 4 have been plotted in different axes, related by a proportionality factor defined as $\alpha$ in Eq. 3. This value is independent of the actual number of SVs in the component (as both quantities scale linearly with it) and can therefore be used to obtain the SEE rate in application from the experimental HEH cross section value. As introduced above, we have selected the proportionality factor as that for which the experimental cross section and in-flight SEE rate coincide at $3 \mathrm{MeVcm}^{2} / \mathrm{mg}$. In this way the respective rate and cross section curves in the $1-10 \mathrm{MeVcm}^{2} / \mathrm{mg}$ range are contained within a factor of 3 . The proportionality factors defined as $\alpha$ in Eq. 3 relating the experimental and application cases 
of interest were calculated as described above and are shown in Table I. For a given application environment, these factors defer by less than $20 \%$ among the different experimental cases, again manifesting that the respective volume-equivalent LET cross sections follow a similar distribution. In addition, the corresponding results for an SEU-like geometry lead to $\alpha$ factors within $20 \%$ of the values reported for SEL, showing a relatively weak impact of the SV dimensions in the considered range.

In addition it is worth noting that in order to obtain an equivalent heavy ion fluence of $10^{7} \mathrm{~cm}^{-2}$ [21] above an LET of $3(10) \mathrm{MeVcm}^{2} / \mathrm{mg}$, a $230 \mathrm{MeV}$ proton fluence of $1.34 \times$ $10^{12} \mathrm{~cm}^{-2}\left(5.3 \times 10^{12} \mathrm{~cm}^{-2}\right)$ is required, corresponding to a TID value of 72 (281) krad.

TABLE I: $\alpha$-factors in units of $\mathrm{cm}^{-2} \mathrm{day}^{-1}$ relating the considered experimental cross section and application SEE rates for different SV geometries.

\begin{tabular}{c|c|c|c|c}
\hline \multirow{2}{*}{ Geometry } & \multirow{2}{*}{ Test Env. } & Protons & \multicolumn{2}{|c}{ Heavy Ions } \\
\cline { 3 - 5 } & & LEO & GEO & LEO \\
\hline \hline \multirow{3}{*}{ SEL } & CHARM (4) & $6.61 \times 10^{6}$ & $5.59 \times 10^{6}$ & $1.56 \times 10^{6}$ \\
& CHARM (4*) & $7.90 \times 10^{6}$ & $6.68 \times 10^{6}$ & $1.86 \times 10^{6}$ \\
& $230 \mathrm{MeV}$ protons & $7.00 \times 10^{6}$ & $5.88 \times 10^{6}$ & $1.64 \times 10^{6}$ \\
\hline SEU & CHARM (4) & $7.80 \times 10^{6}$ & $6.27 \times 10^{6}$ & $1.85 \times 10^{6}$ \\
\hline
\end{tabular}

\section{RESULTS}

\section{A. Ground Testing for VELA approach}

The selected components for the application of the proposed volume-equivalent LET approach (VELA) are five SRAM memories, four of which are of the $0.18 \mu \mathrm{m}$ technology (tested for SEL) and one of the $0.25 \mu \mathrm{m}$ technology (tested for SEU). Hadron tests were performed using a $230 \mathrm{MeV}$ proton beam at PSI [22], a quasi-monoenergetic $300 \mathrm{MeV}$ neutron beam at RCNP [23] and mixed-field tests at CHARM, yielding the HEH cross sections shown in Table II. The parts tested were of the same reference (or very similar, e.g. 16 Mbit SRAM version as opposed to $8 \mathrm{Mbit}$ ) as those embarked in the Proba-II and Alphasat satellites. Different test environments are considered for completeness and in order to exemplify different possible ground level hadron test options, however only a single experimental cross section value in a hadron beam/mixed-field is needed to estimate the in-flight SEE data through the VELA approach.

We then use the CHARM values in Table II together with the factors in Table I to retrieve the expected in-flight SEE rate for the Proba-II and Alphasat experiments through Eq. 3. The resulting values are shown in Table III and will be compared to those derived from the traditional IRPP approach and the actual in-flight values in the next sections.

\section{B. IRPP SEE rate prediction and comparison with VELA}

The proton and heavy ion SEE rates were calculated using the OMERE tool [24]. As an input, the fits of the proton and heavy ion test data [25] were provided and the in-flight SEE rates were calculated for each environment using the integral rectangular parallelepiped (IRPP) method, for which the Weibull fits of the proton and heavy ion test data are
TABLE II: Ground test SEE HEH cross sections in units of $\mathrm{cm}^{2}$ for different components and facilities. For CHARM, the number in brackets represents the test location and the asterisk describes the configuration with shielding (see Fig. 1).

\begin{tabular}{c|c|c|c|c}
\hline SRAM & \multirow{2}{*}{ PSI } & \multirow{2}{*}{ RCNP } & CHARM & CHARM \\
\cline { 4 - 5 } & & & $(4)$ & $\left(4^{*}\right)$ \\
\hline \hline IS61LV5128AL-12 & $2.3 \times 10^{-8}$ & $2.9 \times 10^{-8}$ & - & $1.2 \times 10^{-8}$ \\
BS62LV8001EIP55 & - & $6.4 \times 10^{-8}$ & - & $2.2 \times 10^{-8}$ \\
AS7C34098 & $3.4 \times 10^{-10}$ & - & $1.7 \times 10^{-10}$ & - \\
K6R4008V1D & $4.4 \times 10^{-10}$ & - & $2.6 \times 10^{-10}$ & - \\
AT60142F & $4.4 \times 10^{-7}$ & - & $5.0 \times 10^{-7}$ & - \\
\hline
\end{tabular}

TABLE III: Expected proton and heavy ion SEE rate in units of events per day per device derived using the volume-equivalent LET approach through the $\alpha$-factors and cross sections for CHARM shown in Tables I and II.

\begin{tabular}{c|c|c|c}
\hline \multirow{2}{*}{ SRAM } & Protons & \multicolumn{2}{|c}{ Heavy Ions } \\
\cline { 2 - 4 } & LEO & GEO & LEO \\
\hline IS61LV5128AL-12 & $9.48 \times 10^{-2}$ & $8.02 \times 10^{-2}$ & $2.23 \times 10^{-2}$ \\
BS62LV8001EIP55 & $1.74 \times 10^{-1}$ & $1.47 \times 10^{-1}$ & $4.09 \times 10^{-2}$ \\
K6R4008V1D & $1.72 \times 10^{-3}$ & $1.45 \times 10^{-3}$ & $4.06 \times 10^{-4}$ \\
AS7C34096A & $1.12 \times 10^{-3}$ & $9.50 \times 10^{-4}$ & $2.65 \times 10^{-4}$ \\
AT60142F & 3.87 & 3.11 & 0.92 \\
\hline
\end{tabular}

convoluted with the proton energy and heavy ion LET spectra respectively. Maximum solar conditions were used, and a sensitive volume thickness of 1 and $2 \mu \mathrm{m}$ was considered respectively for SEU and SEL. The selected shielding values were 6 and $10 \mathrm{~mm}$ of aluminum for Alphasat and Proba-II respectively. The data used for the Weibull fits were for a ground testing temperature of $45^{\circ} \mathrm{C}$ for Alphasat (temperature of the satellite in geostationary orbit is roughly $24^{\circ} \mathrm{C}$ ) and $60^{\circ} \mathrm{C}$ for Proba-II (average board temperature in-flight of roughly $50^{\circ} \mathrm{C}$ ). The resulting calculated SEE rates are shown in Table IV including the ratio between then VELA and IRPP prediction values.

TABLE IV: IRPP SEE rate prediction in units of events per component and day. The ratio between the VELA calculation at CHARM (see Table III) and the IRPP value is shown in brackets.

\begin{tabular}{c|c|c}
\hline \multirow{2}{*}{ SRAM } & \multicolumn{2}{|c}{ IRPP predicted SEE rate } \\
\cline { 2 - 3 } & Proba-II (LEO) & Alphasat (GEO) \\
\hline \hline IS61LV5128AL-12 & $1.81 \times 10^{-1}(\mathbf{0 . 6 5})$ & $6.42 \times 10^{-2}(\mathbf{1 . 2 5})$ \\
BS62LV8001EIP55 & - & $4.89 \times 10^{-1}(\mathbf{1 . 7 8})$ \\
K6R4008V1D & $2.57 \times 10^{-3}(\mathbf{0 . 8 3})$ & $5.63 \times 10^{-4}(\mathbf{2 . 5 8})$ \\
AS7C34096A & $1.36 \times 10^{-3}(\mathbf{1 . 0 2})$ & - \\
AT60142F & $2.8(\mathbf{1 . 7 1})$ & $2.7(\mathbf{1 . 1 5})$ \\
\hline
\end{tabular}

As can be seen, the volume-equivalent LET approach SEE rate predictions are within a factor of 3 of the standard IRPP approach for all 5 components considered in both a proton and heavy ion dominated environment. We underline at this stage that whereas the traditional IRPP method requires testing in a proton and heavy ion facility using a relatively broad energy and LET interval, predictions from CHARM are based on a single hadron SEE cross section measurement for each component, which can be performed in parallel for different references provided the appropriate setup is available.

\section{In-flight data and comparison with VELA}

In-flight data for the two missions considered in this paper were presented in [24]. The Proba-II mission was launched on 
November 2, 2009 and occupies a LEO orbit of 713-733 km altitude and 98 degrees of inclination. Its Technology Demonstration Module (TDM) hosts an SEL experiment consisting of four different memories, three of which are considered in the present work. The Proba-2 TDM SEL results for the selected components correspond to 2223 days of in-flight analysis (March 2010 - March 2016).

The Alphasat satellite was launched on July 25, 2013 and occupies a geostationary orbit. It hosts an SEL experiment consisting of four different types of memories, three of which are considered in the present work. For each memory reference there are three different samples. In-flight results here reported correspond to the 28/08/2013-30/09/2015 period for SEL and 02/10/2013-31/08/2015 for SEU. A picture of the Alphasat Memory Test Board (MTB) can be seen in Figure 5.
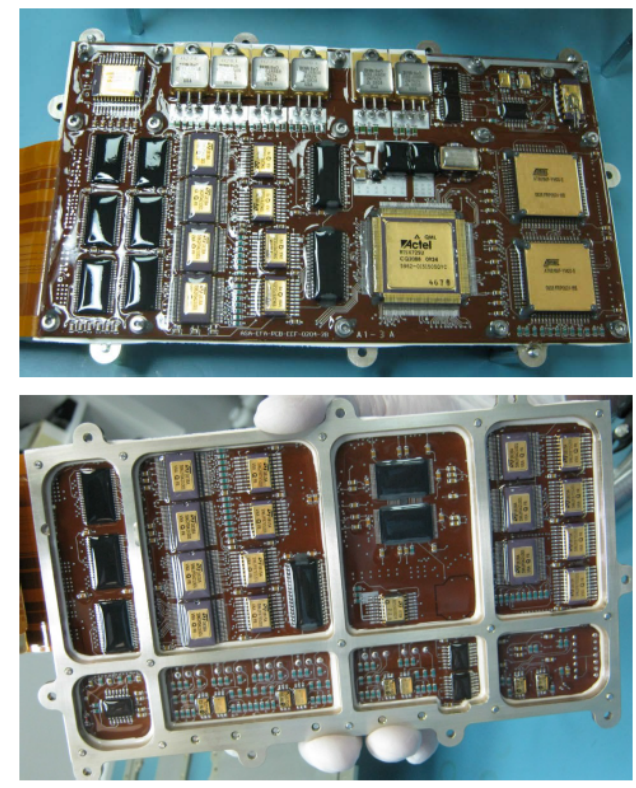

Fig. 5: Picture of the Alphasat Memory Test Board (MTB) hosting among others the SEU and SEL experiments used to extract the inflight data presented in this paper.

The SEE in-flight data for the two different missions and considered memories are reported in Table $\mathrm{V}$ together with the ratio between the values predicted by the VELA approach and the in-flight data. It is to be noted that for the K6R4008V1D component, the number of SEL counts in the Proba-II and Alphasat mission were respectively 8 and 4 . Likewise, the ProbaII SEL count for the AS7C34096A part was 3. Therefore, for these cases the in-flight SEL rates have large associated statistical uncertainties.

TABLE V: In-flight SEL rate in units of events per component and day. The ratio between the volume-equivalent LET approach prediction and the in-flight value is show in brackets.

\begin{tabular}{c|c|c}
\hline \multirow{2}{*}{ SRAM } & \multicolumn{2}{|c}{ In-flight SEL Rate } \\
\cline { 2 - 3 } & Proba-II & Alphasat \\
\hline \hline IS61LV5128AL-12 & $1.98 \times 10^{-1}(\mathbf{0 . 6 5})$ & $4.10 \times 10^{-2}(\mathbf{1 . 2 5})$ \\
BS62LV8001EIP55 & - & $1.96 \times 10^{-1}(\mathbf{4 . 4 3})$ \\
K6R4008V1D & $4.52 \times 10^{-3}(\mathbf{0 . 4 7})$ & $1.96 \times 10^{-3}(\mathbf{0 . 7 4})$ \\
AS7C34096A & $1.70 \times 10^{-3}(\mathbf{0 . 8 2})$ & - \\
AT60142F & $1.8(\mathbf{2 . 6 6})$ & $0.71(\mathbf{4 . 3 9})$ \\
\hline
\end{tabular}

As can be seen, the overall agreement between the VELA calculated values at CHARM and the in-flight results are within a factor of 5. If we exclude the GEO results for AT60142F and BS62LV8001EIP55 (for which the standard IRPP approach also overestimates the observed value by a similar proportion) the agreement is within a factor of 3 . When taking into account uncertainty sources such as the ground level test temperature as compared to the in-flight value, or the error associated to the inter-lot sensitivity spread, we consider these results as highly satisfactory for the proposed screening approach of candidate components.

\section{VELA dependence on hadron energy and SV geometry and materials}

The VELA approach is introduced as a means of screening COTS components for their use in space in a cost and time efficient manner. Therefore, it relies on general assumptions regarding the SV dimensions and surrounding materials. In addition, as shown in Table I, for the SV geometries and test conditions considered, the $\alpha$-factors for a given environment are relatively constant. This is mainly due to the fact that, as has previously been shown [26] above roughly $50 \mathrm{MeV}$ the production of fragments in hadron-silicon interactions with an LET large enough to cause SEE is fairly independent of the incident hadron energy.

However, components with relatively high LET threshold and high-Z materials near the SV are known to have a strong dependence with hadron energy up to roughly $3 \mathrm{GeV}$ owing to the increased fission probability [5], [27]-[29]. The impact of the high- $Z$ energy dependence on the VELA calculated SEE rate value for an interplanetary GCR environment such as that of the Alphasat mission is shown in Fig. 6. The cross section as a function of LET is shown for two different energies, both with and without a $120 \mathrm{~nm}$ thick tungsten layer $200 \mathrm{~nm}$ above the SV. This tungsten density corresponds to roughly $0.5 \mu \mathrm{m}^{3}$ per SRAM cell, which was the amount found in the M1 and connectors in a Scanning Electron Microscope (SEM) analysis of the K6R4008V1D component.

As can be seen, for the pure silicon cases, the estimated SEL cross section as a function of the volume-equivalent LET threshold hardly varies between $230 \mathrm{MeV}$ and $1 \mathrm{GeV}$. However, the impact of the high- $\mathrm{Z}$ material is very different, with an increased cross section for the larger energy case at volumeequivalent LET values of above roughly $20 \mathrm{MeVcm}^{2} / \mathrm{mg}$. For such situations, the monoenergetic value or hardness of the HEH field can have a significant impact on the predicted VELA SEE rate, with more energetic values leading to larger (and probably more realistic) GCR heavy ion induced SEE rate predictions. However, as the high- $\mathrm{Z}$ material volume and location with respect to the $\mathrm{SV}$ in is general unknown, this effect cannot be taken into account explicitly in the model in a systematic way.

In addition, as stated in [1], one of the main constraints of correlating heavy ion SEE rates with hadron experimental data is that for thicker volumes, the volume-equivalent LET distribution falls off much earlier, as the average ranges of the high-LET products become shorter than the SV characteristic 


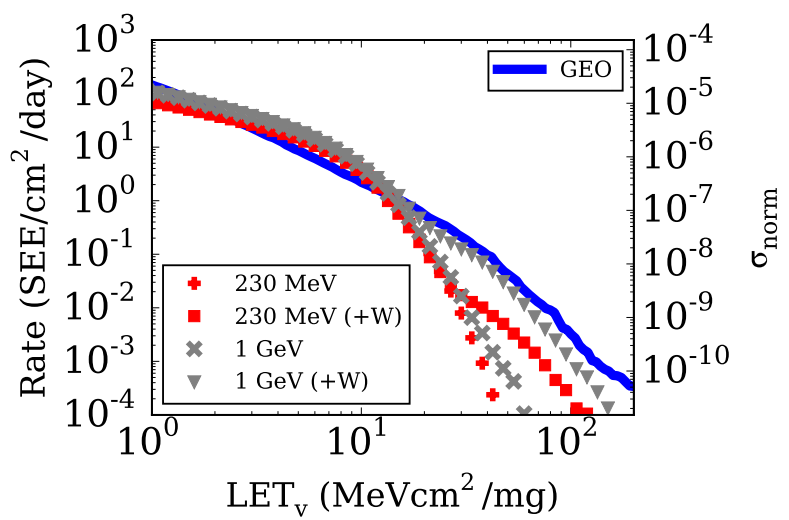

Fig. 6: Simulated SEE cross section and rate normalized to the SV surface for the SEL model both with a pure silicon geometry and a layout containing a $120 \mathrm{~nm}$ tungsten layer $200 \mathrm{~nm}$ above the SV.

dimensions. As a consequence the deposited energy is roughly the same, thus resulting in a decreased volume-equivalent LET value. This effect is illustrated in Fig. 7, in which the geostationary GCR SEE rate and experimental $230 \mathrm{MeV}$ proton cross sections are plotted as a function of the volumeequivalent LET for SV thicknesses of 2 and $10 \mu \mathrm{m}$. As can be seen, the different depth values result in changes in both the GCR rate and experimental proton cross sections in a nonproportional way: whereas at around $10 \mathrm{MeVcm}^{2} / \mathrm{mg}$ the 2 $\mu \mathrm{m}$ experimental curve still mimics the GCR one, for $10 \mu \mathrm{m}$ this is clearly not the case.

This effect is further shown in Fig. 8, in which the $\alpha$ factor is plotted as a function of SV thickness for two different volumeequivalent LET threshold values. As can be seen, whereas for low LET $_{\mathrm{v}}$ threshold values the $\alpha$ factor is relatively stable in a broad thickness range, for large $\mathrm{LET}_{\mathrm{v}}$ threshold values and thick SVs $(\sim 10 \mu \mathrm{m})$ the value of $\alpha$ rapidly diverges, becoming strongly dependent on both parameters. In fact, the alpha value relating the experimental hadron cross section and the application SEE rate will tend to infinity for volumeequivalent LET values which are not reached in the hadron tests but are present in the GCR environment, as is described in Fig. 7. This risk of having a volume-equivalent LET threshold in this range and thus overestimating the radiation hardness of the device through hadron testing increases with increasing SV thickness values. Therefore as will be further developed in Section V, these components are an example of cases which would require heavy ion testing after having passed the hadron per-screening.

In addition, we further underline at this stage that whereas a systematic analysis of the typical SEL thickness values as a function of CMOS technology is beyond the scope of this paper, the value of $2 \mu \mathrm{m}$ considered here as based on laser experiments and successful in predicting the HI SEL inflight rate based on hadron tests should not be applied in a generalized way. As a counter-example, the AD9240 $0.6 \mu \mathrm{m}$ bulk silicon ADC reported in [30] is shown to have an SEL collection depth of at least $20 \mu \mathrm{m}$ based on the comparison of test data with ions of similar LET but different range. For such a thickness value, the risk of overestimating the corresponding

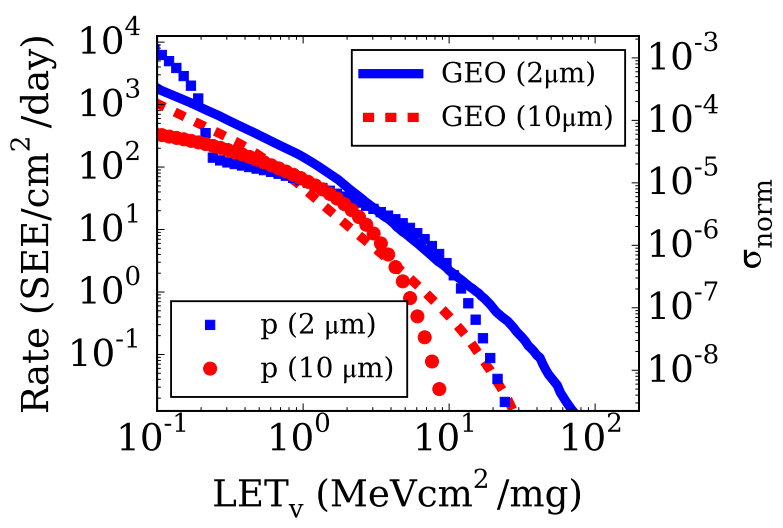

Fig. 7: Simulated SEE rate and experimental $230 \mathrm{MeV}$ proton cross section as a function of the volume-equivalent LET for SEL SV thickness values of 2 and $10 \mu \mathrm{m}$.

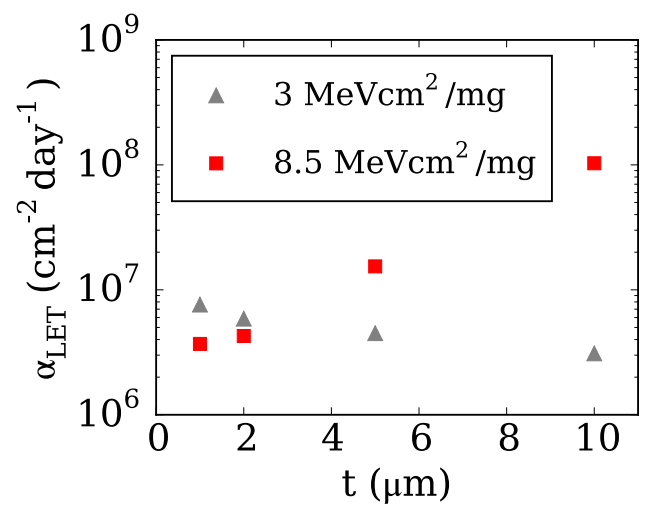

Fig. 8: Heavy ion $\alpha$-factor as a function of SV thickness for a $20 \mathrm{x} 4$ $\mu \mathrm{m}^{2} \mathrm{SV}$ surface and two different volume-equivalent LET threshold values.

HI hardness through hadron testing is large. Therefore, caution is warranted with respect to the SEL SV thickness assumption.

\section{RADIATION HARDNESS ASSURANCE IMPLICATIONS}

As introduced in Section III and applied in Section IV, the volume-equivalent LET approach can be used to estimate the in-flight SEE rate for both the trapped proton and near-Earth interplanetary GCR environments based on single ground level hadron test results. Based on these test results, components can be screened and rejected if considered too sensitive. When compared to the standard IRPP qualification, the proposed approach has the advantage of requiring a single measurement per component as opposed to a set of heavy ion and proton data in a relatively broad LET and energy range.

In addition to being cost and time efficient, the proposed screening approach has the practical advantage of not requiring opening the part. This can be particularly useful for component architectures such as e.g. flip-chips for which enabling access to the SV for standard ground level heavy ion energies (< $10 \mathrm{MeV} / \mathrm{n}$ ) can be particularly challenging [31].

Moreover, at CHARM and as opposed to standard monoenergetic hadron beams, a large number of components can be tested in parallel. This is particularly useful when having to 
characterize a broad range of references (e.g. electrically compatible parts from different manufacturers with a potentially very different sensitivity to SEEs) or the inter-lot and intra-lot sensitivity spread for a particular reference.

It is important to note however that in order to mitigate the risk associated to an underestimation of the SEE HI rates from hadron experiments (e.g. for devices and effects with thick SVs and a high LET threshold) critical parts passing the hadron prescreening should be selected of a full HI characterization. This is particularly true for components with thick SVs and large LET threshold values for which the risk of underestimating the GCR heavy ion SEE rate through the VELA approach is high.

In addition, by assuming a certain value for the saturation cross section of the part (e.g. its full surface area as worst-case) a test in a hadron field yielding zero errors for a given $\mathrm{HEH}$ fluence can be used to determine the associated lower limit of the $\mathrm{LET}_{\mathrm{V}}$ threshold and corresponding upper limit in terms of expected in-flight SEE rate, which might be considered as low enough to accept the part based on the screening without the need of further testing for non-critical components. However it is to be noted that also in this case the upper limit will depend on the assumed SV depth.

Moreover, it is important to note that whereas the interplanetary volume-equivalent LET distribution is generated by high energy ions (with large fluxes up to $Z=28$ and peaking roughly at an energy of $500 \mathrm{MeV} / \mathrm{n}$ ) the CHARM and $230 \mathrm{MeV}$ proton cases are generated by silicon recoils and fragments with considerably lower energies (e.g. as shown in [32], only $1 \%$ of the ${ }^{6} \mathrm{C}$ fragments generated in $230 \mathrm{MeV}$ proton interactions with silicon have an energy above roughly 2 $\mathrm{MeV} / \mathrm{n}$ ). The possible implications of such differences (e.g. ionization track structure, nuclear reactions) on the resulting SEE rate estimation need to be carefully considered.

\section{Conclusions And Outlook}

The in-flight benchmark of the proposed SEE rate estimation approach shows that the latter can be successfully applied in the case of the components and missions considered. The agreement level is comparable to the standard characterization approach, requiring a much more complex ground test methodology. Despite its limitations (mainly related to underestimating the in-flight heavy ion SEE rate for components with high LET threshold and thick SVs with respect to the typical heavy fragment ranges) its simplicity in terms of the amount of measurement required makes it a promising approach to perform low-cost, rapid measurements mainly focused on screening parts and rejecting those considered too sensitive. Parts passing the test can either be accepted with a certain upper limit dependent on the considered SV thickness or selected for HI testing in order to more accurately determine the respective error rate.

The proposed approach was shown to be valid for a set of SEU and SEL results (typically involving smaller SVs) but is expected to break down for SEEs with larger SVs (e.g. SEB) as the volume-equivalent LET curves produced by hadrons fail to reproduce the environmental case.
Moreover, the CELESTA cubesat mission [33], [34] developed in collaboration between CERN and the University of Montpellier will host an experimental SEE payload characterized at CHARM and aimed at extending the volume-equivalent LET approach benchmark to a broader set of components and effects.

\section{REFERENCES}

[1] R. Ladbury, J.-M. Lauenstein, and K. P. Hayes, "Use of proton SEE data as a proxy for bounding heavy-ion SEE susceptibility," IEEE Trans. Nucl. Sci., vol. 62, no. 6, pp. 2505-2510, Dec 2015.

[2] P. M. O'Neill, G. D. Badhwar, and W. X. Culpepper, "Risk assessment for heavy ions of parts tested with protons," IEEE Transactions on Nuclear Science, vol. 44, no. 6, pp. 2311-2314, Dec 1997.

[3] R. G. Alia, "Radiation fields in high energy accelerators and their impact on single event effects," Ph.D. dissertation, Universite Montpellier 2, Dec. 2013. [Online]. Available: http://cds.cern.ch/record/2012360

[4] J. Mekki, M. Brugger, R. Alia, A. Thornton, N. Dos Santos Mota, and S. Danzeca, "A mixed field facility at CERN for radiation test: CHARM," in Radiation and Its Effects on Components and Systems (RADECS), 2015 15th European Conference on, Sept 2015, pp. 284-287.

[5] R. G. Alia, M. Brugger, S. Danzeca, V. Ferlet-Cavrois, C. Frost, R. Gaillard, J. Mekki, F. Saigne, A. Thornton, S. Uznanski, and F. Wrobel, "SEL hardness assurance in a mixed radiation field," IEEE Trans. Nucl. Sci., vol. 62, no. 6, pp. 2555-2562, Dec 2015.

[6] A. Thornton, "CHARM Facility Test Area Radiation Field Description," Apr 2016. [Online]. Available: http://cds.cern.ch/record/2149417

[7] K. Roed, V. Boccone, M. Brugger, A. Ferrari, D. Kramer, E. Lebbos, R. Losito, A. Mereghetti, G. Spiezia, and R. Versaci, "FLUKA simulations for SEE studies of critical LHC underground areas," IEEE Trans. Nucl. Sci., vol. 58, no. 3, pp. 932 -938, June 2011.

[8] K. Roed, M. Brugger, D. Kramer, P. Peronnard, C. Pignard, G. Spiezia, and A. Thornton, "Method for measuring mixed field radiation levels relevant for SEEs at the LHC," IEEE Trans. Nucl. Sci., vol. 59, no. 4, pp. $1040-1047$, Aug. 2012.

[9] A. Ferrari, P. R. Sala, A. Fasso, and J. Ranft, "FLUKA, a multiparticle transport code," CERN-2005-10-2005, INFN/TC-05/11, SLAC$R-773,2005$.

[10] T. Böhlen, F. Cerutti, M. Chin, A. Fassò, A. Ferrari, P. Ortega, A. Mairani, P. Sala, G. Smirnov, and V. Vlachoudis, "The FLUKA code: developments and challenges for high energy and medical applications," Nuclear Data Sheets, vol. 120, pp. 211-214, 2014.

[11] G. Battistoni, T. Boehlen, F. Cerutti, P. W. Chin, L. S. Esposito, A Fass, A. Ferrari, A. Lechner, A. Empl, A. Mairani, A. Mereghetti, P. G. Ortega, J. Ranft, S. Roesler, P. R. Sala, V. Vlachoudis, and G. Smirnov, "Overview of the FLUKA code," Annals of Nuclear Energy, vol. 82, pp. $10-18,2015$.

[12] J. L. Barth, C. S. Dyer, and E. G. Stassinopoulos, "Space, atmospheric, and terrestrial radiation environments," IEEE Trans. Nucl. Sci., vol. 50, no. 3, pp. 466-482, June 2003.

[13] A. J. Tylka, J. H. Adams, P. R. Boberg, B. Brownstein, W. F. Dietrich, E. O. Flueckiger, E. L. Petersen, M. A. Shea, D. F. Smart, and E. C. Smith, "CREME96: A revision of the cosmic ray effects on microelectronics code," IEEE Trans. Nucl. Sci., vol. 44, no. 6, pp. 2150-2160, 1997.

[14] B. D. Sierawski, J. A. Pellish, R. A. Reed, R. D. Schrimpf, K. M. Warren, R. A. Weller, M. H. Mendenhall, J. D. Black, A. D. Tipton, M. A Xapsos, R. C. Baumann, X. Deng, M. J. Campola, M. R. Friendlich, H. S. Kim, A. M. Phan, and C. M. Seidleck, "Impact of low-energy proton induced upsets on test methods and rate predictions," IEEE Trans. Nucl. Sci., vol. 56, no. 6, pp. 3085-3092, Dec 2009.

[15] "OMERE software TRAD tests and radiations." [Online]. Available: http://www.trad.fr/OMERE-Software.html

[16] E. Faraud, V. Pouget, K. Shao, C. Larue, F. Darracq, D. Lewis, A. Samaras, F. Bezerra, E. Lorfevre, and R. Ecoffet, "Investigation on the SEL sensitive depth of an SRAM using linear and two-photon absorption laser testing," IEEE Trans. Nucl. Sci., vol. 58, no. 6, pp. 2637-2643, Dec. 2011.

[17] F. Bezerra, E. Lorfevre, R. Ecoffet, D. Peyre, C. Binois, S. Duzellier, D. Falguere, T. Nuns, M. Melotte, P. Calvel, R. Marec, N. Chatry, W. Falo, and C. Deneau, "In flight observation of proton induced destructive single event phenomena," in Radiation and Its Effects on Components and Systems (RADECS), 2009 European Conference on, Sept. 2009, pp. 126-132. 
[18] Y.-T. Yu, J.-W. Han, G.-Q. Feng, M.-H. Cai, and R. Chen, "Correction of single event latchup rate prediction using pulsed laser mapping test," IEEE Trans. Nucl. Sci., vol. 62, no. 2, pp. 565-570, April 2015.

[19] R. A. Weller, M. H. Mendenhall, R. A. Reed, R. D. Schrimpf, K. M. Warren, B. D. Sierawski, and L. W. Massengill, "Monte Carlo simulation of single event effects," IEEE Trans. Nucl. Sci., vol. 57, no. 4, pp. 17261746, 2010.

[20] A. Infantino, R. G. Alia, and M. Brugger, "Monte Carlo evaluation of single event effects in a deep-submicron bulk technology: comparison between atmospheric and accelerator environment," IEEE Transactions on Nuclear Science, vol. PP, no. 99, pp. 1-1, 2016.

[21] Single Event Effects Test Method and Guidelines. ESCC Basic Specification No. 25100, 2014.

[22] W. Hajdas, F. Burri, C. Eggel, R. Harboe-Sorensen, and R. deMarino, "Radiation effects testing facilities in PSI during implementation of the Proscan project," in Radiation Effects Data Workshop, 2002 IEEE, 2002, pp. $160-164$.

[23] Y. Iwamoto, M. Hagiwara, D. Satoh, S. Araki, H. Yashima, T. Sato, A. Masuda, T. Matsumoto, N. Nakao, T. Shima et al., "Characterization of high-energy quasi-monoenergetic neutron energy spectra and ambient dose equivalents of 80-389 $\mathrm{MeV} 7 \mathrm{Li}(\mathrm{p}, \mathrm{n})$ reactions using a time-offlight method," Nuclear Instruments and Methods in Physics Research Section A: Accelerators, Spectrometers, Detectors and Associated Equipment, vol. 804, pp. 50-58, 2015.

[24] C. Poivey, "Update on PROBA-2 TDM and ALPHASAT TDP8 Flight Experiments on Memories," in Single Event Effects Symposium, May 2016.

[25] R. Harboe-Sorensen, C. Poivey, N. Fleurinck, K. Puimege, A. Zadeh, F.-X. Guerre, F. Lochon, M. Kaddour, L. Li, D. Walter, A. Keating, A. Jaksic, and M. Poizat, "The technology demonstration module on-board Proba-II," IEEE Trans. Nucl. Sci., vol. 58, no. 3, pp. 1001 -1007, June 2011.

[26] D. M. Hiemstra and E. W. Blackmore, "LET spectra of proton energy levels from 50 to $500 \mathrm{MeV}$ and their effectiveness for single event effects characterization of microelectronics," IEEE Trans. Nucl. Sci., vol. 50, no. 6, pp. 2245-2250, Dec. 2003.

[27] J. R. Schwank, M. R. Shaneyfelt, J. Baggio, P. E. Dodd, J. A. Felix V. Ferlet-Cavrois, P. Paillet, D. Lambert, F. W. Sexton, G. L. Hash, and E. W. Blackmore, "Effects of particle energy on proton-induced singleevent latchup," IEEE Trans. Nucl. Sci., vol. 52, no. 6, pp. $2622-2629$, Dec. 2005.

[28] R. G. Alia, E. W. Blackmore, M. Brugger, S. Danzeca, V. FerletCavrois, R. Gaillard, J. Mekki, C. Poivey, K. Roed, F. Saigne, G. Spiezia, M. Trinczek, S. Uznanski, and F. Wrobel, "SEL cross section energy dependence impact on the high energy accelerator failure rate," IEEE Trans. Nucl. Sci., vol. 61, no. 6, pp. 2936-2944, Dec 2014.

[29] T. L. Turflinger, D. A. Clymer, L. W. Mason, S. Stone, J. S. George, M. Savage, R. Koga, E. Beach, and K. Huntington, "RHA implications of proton on gold-plated package structures in SEE evaluations," IEEE Trans. Nucl. Sci., vol. 62, no. 6, pp. 2468-2475, Dec 2015.

[30] T. F. Miyahira, A. H. Johnston, H. N. Becker, S. D. LaLumondiere, and S. C. Moss, "Catastrophic latchup in cmos analog-to-digital converters," IEEE Transactions on Nuclear Science, vol. 48, no. 6, pp. 1833-1840, Dec 2001.

[31] H. Quinn, "Challenges in testing complex systems," IEEE Trans. Nucl. Sci., vol. 61, no. 2, pp. 766-786, April 2014.

[32] R. G. Alia, M. Brugger, S. Danzeca, V. Ferlet-Cavrois, C. Poivey, K. Roed, F. Saigne, G. Spiezia, S. Uznanski, and F. Wrobel, "SEE measurements and simulations using mono-energetic GeV-energy hadron beams," IEEE Trans. Nucl. Sci., vol. 60, no. 6, pp. 4142-4149, Dec. 2013.

[33] R. Secondo, P. Peronnard, A. Merlenghi, M. Brugger, E. Chesta, S. Danzeca, , R. Garcia Alia, A. Masi, M. Bernard, L. Dusseau, and J. R. Vaille, "A nanosatellite radiation qualification procedure at the CHARM facility based on the CELESTA payload demonstrator," 4S Symposium 2016, submitted for publication.

[34] A. Merlenghi, M. Brugger, E. Chesta, S. Danzeca, , R. Garcia Alia, A. Masi, P. Peronnard, R. Secondo, M. Bernard, and L. Dusseau, "The CELESTA cubesat radiation monitoring mission ," 4 S Symposium 2016, submitted for publication. 